\title{
Repetitive Transcranial Magnetic Stimulation for Wernicke-Korsakoff Syndrome: A Case Report
}

\author{
So Won Chung, $\mathrm{MD}^{1}$, Shin Who Park, $\mathrm{MD}^{1}$, Young Jae Seo, $\mathrm{MD}^{1}$, \\ Jae-Hyung Kim, MD, PhD ${ }^{1}$, Chan Ho Lee, MD $^{2}$, Jong Youb Lim, MD ${ }^{1}$
}

${ }^{1}$ Department of Rehabilitation Medicine, Eulji University Hospital, Eulji University School of Medicine, Daejeon; ${ }^{2}$ Department of Rehabilitation Medicine, Eulji Hospital, Eulji University School of Medicine, Seoul, Korea

\begin{abstract}
A 57-year-old man who was diagnosed with Wernicke-Korsakoff syndrome showed severe impairment of cognitive function and a craving for alcohol, even after sufficient supplementation with thiamine. After completing 10 sessions of $10 \mathrm{~Hz}$ repetitive transcranial magnetic stimulation (rTMS) at 100\% of the resting motor threshold over the left dorsolateral prefrontal cortex, dramatic improvement in cognitive function and a reduction in craving for alcohol were noted. This is the first case report of the efficacy of a high-frequency rTMS in the treatment of Wernicke-Korsakoff syndrome.
\end{abstract}

Keywords Wernicke-Korsakoff syndrome, Repetitive transcranial magnetic stimulation

\section{INTRODUCTION}

Wernicke-Korsakoff syndrome (WKS) is a disorder of the brain resulting from thiamine deficiency, and it is commonly associated with chronic alcoholism. WKS is a neuropathological term which encompasses two separate conditions-Wernicke encephalopathy (WE) and Korsakoff syndrome (KS). The classic clinical triad of $\mathrm{WE}$ is ophthalmoplegia, ataxia, and confusion, and KS is characterized by severe impairment of current and recent memory. WE is reversible if treated in a timely manner with thiamine administration but if it is undiagnosed or inadequately treated, it is likely to progress to the chronic state, KS [1]. The best treatment for KS is known as early recognition and management of $\mathrm{WE}$, and the optimal treatment for KS itself is not yet clear [2].

Repetitive transcranial magnetic stimulation (rTMS) is a noninvasive brain stimulation technique which modulates cortical excitability and it is proven to have therapeutic effects in various neuropsychiatric disorders [3].

Received May 31, 2016; Accepted July 6, 2016

Corresponding author: Jong Youb Lim

Department of Rehabilitation Medicine, Eulji University Hospital, 95 Dunsanseo-ro, Seo-gu, Daejeon 35233, Korea. Tel: +82-42-611-3631, Fax: +82-42611-3633, E-mail: jylimmd@eulji.ac.kr

ORCID: So Won Chung (http://orcid.org/0000-0003-2344-2832); Shin Who Park (http://orcid.org/0000-0002-7136-9644); Young Jae Seo (http://orcid. org/0000-0003-3791-5012); Jae-Hyung Kim (http://orcid.org/0000-0002-7005-0113); Chan Ho Lee (http://orcid.org/0000-0002-5129-6652); Jong Youb Lim (http://orcid.org/0000-0001-9100-4864).

@ This is an open-access article distributed under the terms of the Creative Commons Attribution Non-Commercial License (http://creativecommons.org/ licenses/by-nc/4.0) which permits unrestricted noncommercial use, distribution, and reproduction in any medium, provided the original work is properly cited. Copyright $\odot 2017$ by Korean Academy of Rehabilitation Medicine 
In addition, previous studies have demonstrated the anticraving effects of rTMS in substance dependence [4].

We expected rTMS would be a useful treatment tool for enhancing cognitive function and suppressing co-occurring alcohol cravings in WKS. We herein report a case of a WKS patient who showed dramatic improvement in cognitive function and reduction in craving for alcohol following high-frequency rTMS.

\section{CASE REPORT}

A 57-year-old man visited our emergency center presenting with dyspnea which started 4 days ago. He was admitted to the pulmonary department of Eulji University Hospital with a diagnosis of tuberculous pleurisy. He had a 4-year history of diabetes mellitus, hypertension and a 30-year history of heavy alcohol abuse. He had consumed a bottle of soju every day, and in the last 6 months, he had been drinking 5 bottles of soju daily with poor diet. His family history was unremarkable, and laboratory examinations on admission showed leukocytosis $(12,380 /$ $\left.\mathrm{mm}^{3}\right)$, elevated C-reactive protein $(4.50 \mathrm{mg} / \mathrm{dL})$, hyponatremia (128 mEq/L), hypoalbuminemia $(2.8 \mathrm{~g} / \mathrm{dL})$, abnormal liver function (aspartate transaminase $98 \mathrm{IU} / \mathrm{L}$ and alanine transaminase $37 \mathrm{IU} / \mathrm{L}$ ), and a plasma glucose level of $130 \mathrm{mg} / \mathrm{dL}$. He showed a confused mentality and his wife revealed that he had been unable to walk for 10 days, complaining of weakness in the lower extremities. A manual muscle test checked with the Medical Research Council scale showed $2 / 2$ for the lower extremities.

He was consulted to the neurology department because his confused mentality, attention deficits and weakness in the lower extremities did not show improvement. At the initial evaluation, the patient kept telling a confabulated story about his deceased father visiting the hospital and showed short-term memory impairment, as he was unable to recall three items after a few minutes. In addition, he could not explain what had happened in the past few days or remember how he has come to the hospital in detail. He had ataxia with dysmetria bilaterally seen on the finger-to-nose test, but ophthalmoplegia was not observed. Brain magnetic resonance imaging (MRI) was done in order to rule out acute cerebrovascular disease and to detect changes in areas which are more vulnerable to thiamine deficiency such as mammillary bodies [5]. There was no definite signal abnormality detected on the MRI (Fig. 1), and peripheral sensorimotor polyneuropathy was observed in a nerve conduction study. Based on the clinical evaluation, he was diagnosed with WKS. At the time of the diagnosis, he was taking medication for tuberculosis. In addition, he received a $100 \mathrm{mg}$ of thiamine intravenously for WKS.

After a month, he was transferred to the rehabilitation department. He still showed a confused mentality, craved not only alcohol but also cigarettes, which he had quit 10 years ago, and he scored 13/30 on the Korean version of Mini-Mental Status Examination (K-MMSE). Pharmacological cognitive enhancement or anti-craving agent other than $200 \mathrm{mg}$ of the B-complex vitamin was not used, and cognitive function training was maintained. Laboratory data showed vitamin $\mathrm{B} 1$ level of $320.3 \mathrm{nmol} / \mathrm{L}$, which was above the normal range (66.1-220.1 nmol/L), but he scored 16/30 at follow-up using the K-MMSE, and no definite clinical improvement was observed.

In order to evaluate baseline cognitive function, we performed following tests: K-MMSE, Loewenstein Occupational Therapy Cognitive Assessment (LOTCA), the Korean version of Montreal Cognitive Assessment (MOCA-K), Digit Span subtest of Korean Wechsler Adult Intelligence Scale-IV (K-WAIS-IV), and Rey-Osterrieth Complex Figure Test (ROCFT). To measure the severity of alcohol dependence and baseline craving, the Alcohol Craving Questionnaire-Short Form-Revised (ACQ-SF-R) and

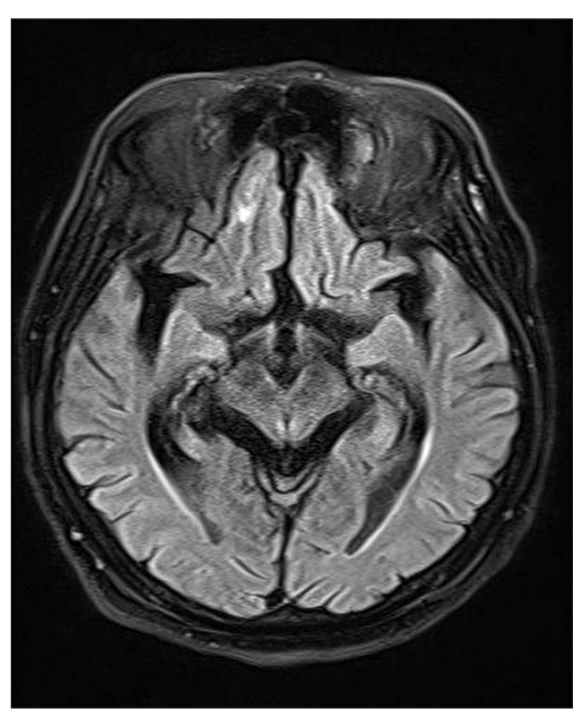

Fig. 1. Axial fluid-attenuated inversion recovery image shows no definite signal change in the mammillary bodies. 
Clinical Institute Withdrawal Assessment for AlcoholRevised (CIWA-Ar) were documented. In addition, the International Cooperative Ataxia Rating Scale (ICARS), Berg Balance Scale (BBS), and hand function tests (HFTs) including Purdue and Grooved Pegboard Tests, and Box and Block Test were done to evaluate ataxia.

We performed 10 sessions of rTMS over 2 weeks in order to investigate the effect of rTMS on cognition, alcohol dependence and ataxia at the same time. The patient was informed of the side effects and risks that he might experience during participation and submitted a written informed consent. Stimulation was applied by a Magstim Rapid2 System (Magstim, Whitland, UK), using a Double $70 \mathrm{~mm}$ Air Film Coil. Each session consisted of 20 trains of 5 seconds' duration separated by 25 seconds' pauses. Stimulation was applied at $10 \mathrm{~Hz}$ frequency and at an intensity of $100 \%$ resting motor threshold (RMT). Total 1,000 pulses were delivered per treatment. RMT was defined as the stimulus intensity that produced visibly observable contractions of the right abductor pollicis brevis at least 5 times out of 10 stimuli. rTMS was performed over the left dorsolateral prefrontal cortex (DLPFC), which was determined by moving the coil $5 \mathrm{~cm}$ anteriorly from the point of RMT determination. No specific side effect was identified during 10 sessions of performance.

We re-assessed K-MMSE, Digit Span subtest of K-WAISIV, ACQ-SF-R, and CIWA-Ar when 5 sessions of treatment were done. Clinically, cognitive function and craving for alcohol were improved with positive changes observed in follow-up K-MMSE and ACQ-SF-R. After completion of 10

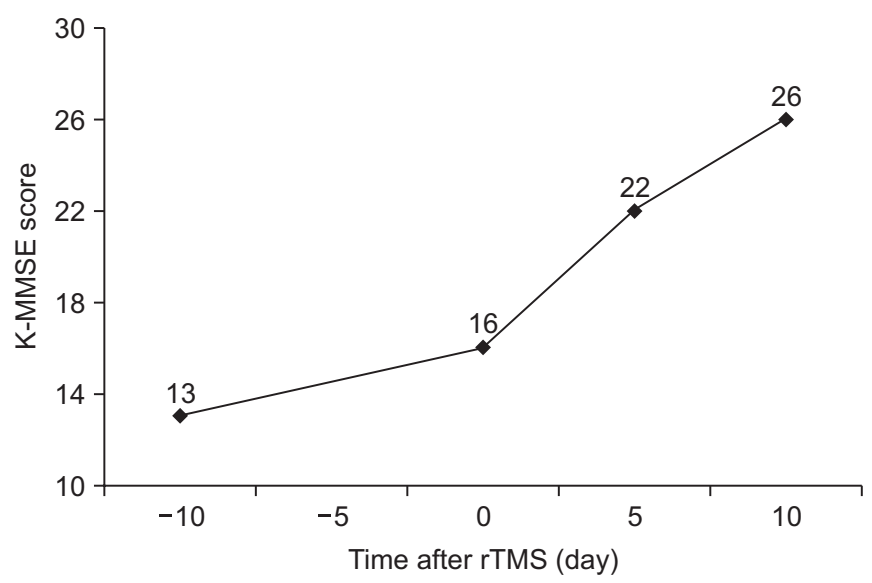

Fig. 2. Change of Korean version of Mini-Mental Status Examination (K-MMSE) scores induced by repetitive transcranial magnetic stimulation (rTMS). sessions, all the aforementioned initial baseline evaluations and questionnaires were re-evaluated. A significant improvement in cognitive function was observed in $\mathrm{K}$ MMSE during the treatment (Fig. 2), as well as improvement observed in parameters of all the other follow-up cognitive function evaluations (Table 1). In respect of alcohol craving, a significant reduction was found in ACQ-SF-R total score, and there were also positive changes observed in ICARS, BBS, and HFTs after the treatment (Table 1).

Table 1. Scores of functional assessments and craving questionnaires at pre-rTMS and post-rTMS

\begin{tabular}{lcc}
\hline & Pre-rTMS & Post-rTMS \\
\hline Cognition & & \\
LOTCA (26-115) & 79 & $111^{\mathrm{a})}$ \\
\hline MOCA-K (0-30) & 14 & $20^{\mathrm{a})}$ \\
\hline DSp K-WAIS-IV (0-48) & 27 & $30^{\mathrm{a})}$ \\
\hline ROCFT-I (0-36) & 0 & $5^{\mathrm{a})}$ \\
\hline ROCFT-D (0-36) & 0 & $2^{\mathrm{a})}$ \\
\hline Craving & & \\
ACQ-SF-R-T (12-84) & 62 & $35^{\mathrm{b})}$ \\
\hline CIWA-Ar (0-67) & 9 & $3^{\mathrm{b})}$ \\
\hline Ataxia & 35 & $32^{\mathrm{b})}$ \\
\hline ICARS (0-100) & 4 & $8^{\mathrm{a})}$ \\
\hline BBS (0-56) & 3 & $5^{\mathrm{a})}$ \\
\hline HFTs & Rt. $>300$ & Rt. 150.12 \\
\hline PPT (/30 s) & Lt $>300$ & Lt. 198.50 \\
\hline GPT (s) & Rt. 37 & Rt. 54 \\
\hline BBT & Lt. 40 & Lt. $40^{\mathrm{a})}$ \\
\hline
\end{tabular}

rTMS, repetitive transcranial magnetic stimulation; LOTCA, Loewenstein Occupational Therapy Cognitive Assessment; MOCA-K, Korean version of Montreal Cognitive Assessment; DSp K-WAIS-IV, Digit Span subtest of Korean Wechsler Adult Intelligence Scale-IV; ROCFT-I, ReyOsterrieth Complex Figure Test-immediate recall test; ROCFT-D, Rey-Osterrieth Complex Figure Test-delayed recall test; ACQ-SF-R-T, Alcohol Craving QuestionnaireShort Form-Revised total score; CIWA-Ar, Clinical Institute Withdrawal Assessment for alcohol-revised; ICARS, International Cooperative Ataxia Rating Scale; BBS, Berg Balance Scale; HFT, hand function test; PPT, Purdue Pegboard Test; GPT, Grooved Pegboard Test; BBT, Box and Block Test.

${ }^{a)}$ Denotes tests in which increment of scores indicates

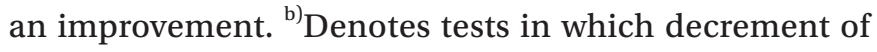
scores indicates an improvement. 


\section{DISCUSSION}

At present, clinical examination is the standard in diagnosing WKS as there is no reliable diagnostic test, and while being helpful, the diagnostic utility of MRI is only in confirming a clinical suspicion because of its low sensitivity [5]. Though WKS is diagnostically challenging, it is important for the physician to have suspicion for patients who are at high risk and administer thiamine promptly following the diagnosis to minimize neuronal death due to thiamine deficiency. The thalamus and mammillary bodies are known to be more vulnerable to thiamine deficiency, and as they play an important role in the neural circuitry of memory, lesions in these areas can cause memory impairment [6]. However, our patient did not show significant improvement in cognitive function, and craving for alcohol and cigarette was increased even with sufficient thiamine administration. Failure of thiamine treatment is thought to be possibly due to irreversibly damaged cells by lack of thiamine, deficiencies of other nutrients or alcohol-induced neurotoxicity [1].

While several cognitive enhancers such as galantamine, atomoxetine and memantine have been reported to be promising for the treatment of alcohol dependence [7], their efficacy is inconclusive to date, and to elicit improvement in a short period of time, we decided to perform rTMS. There is accumulating evidence on rTMS as a treatment tool for cognitive impairment and substance dependence in isolation. Previous studies indicate highfrequency rTMS over the left DLPFC has a positive effect on cognition [3]. rTMS has also been reported to have anti-craving efficacy in various substance abuse disorders including alcohol dependence $[4,8,9]$. The patients with alcohol dependence have been displayed increased activity in the mesolimbic dopaminergic pathway, and the application of rTMS over the DLPFC is thought to have reduced craving by modulating the altered activity in the mesolimbic pathway through the meso-frontolimbic connections $[4,8,9]$. It was demonstrated that high-frequency rTMS over the right DLPFC has a positive effect on decreasing craving score in patients with alcohol dependence and it is presumed to be due to transsynaptic suppression of the left DLPFC via transcallosal connections [8]. However, in a recent study comparing the anti-craving efficacy of rTMS over the right to the left DLPFC showed no significant difference and rTMS over the left DLPFC showed efficacy in severe cases of alcohol dependence compared to the right side [9]. It is considered that rTMS over left DLPFC affects craving circuits directly, whereas stimulation over the right affects it indirectly [9]. Accordingly, we performed high-frequency rTMS over the left DLPFC in WKS aiming to have a dual effect, which is to improve cognitive function and to decrease co-occurring alcohol craving.

Among clinical symptoms of WKS, our patient initially showed confusion, ataxia, memory impairment and craving for alcohol. After completing 10 sessions of rTMS, the improvement was noted in every cognitive function baseline evaluation, especially remarkable improvement in orientation and attention was observed in K-MMSE, MOCA-K, and LOTCA. There was also the noticeable progression of visuospatial perception in MOCA-K and LOTCA, and improvement in visuospatial memory and processing speed was detected in ROCFT. WKS patients have been reported to perform poorly on tests of frontal lobe function, particularly on tests of executive functioning [1]. The DLPFC plays a crucial role in executive function especially in working memory, and the left DLPFC is reported to be mostly related to the performance of verbal working memory [10]. While the neurophysiological mechanisms responsible for rTMS-induced cognitive facilitation remain essentially unknown, in our case, rTMS might have modulated activity in neural circuits that are involved in executive function including working memory, thus leading to improvement in cognitive function. In terms of ataxia, dysmetria which was initially observed in the finger-to-nose test was gone and significant improvement was detected in follow-up HFTs. He was able to feed and dress without assistance which could not be done before the treatment. Since he had severe weakness in the lower extremities to which peripheral sensorimotor polyneuropathy and disuse atrophy may have contributed, he scored poorly in BBS and ICARS from the baseline, and this may be the reason for the minimal change.

Interestingly, after admission, our patient craved not only for alcohol but also for cigarettes which he had quitted 10 years ago, especially in the night time. Though we did not use measures of cigarette craving, after completing 3 sessions of rTMS, reduction in craving for both alcohol and cigarettes was reported from his wife and the sleep-wake cycle began to be regulated. After completing 10 sessions, the patient did not seek for cigarettes any- 
more. As the efficacy of rTMS on attenuating cigarette craving was reported in previous studies [4], we could assume rTMS also had a positive influence on controlling cigarette craving. To reflect the changes in alcohol craving, we used ACQ-SF-R and CIWA-Ar. A significant reduction was found in ACQ-SF-R after completing the treatment. This result is considered meaningful in clinical aspect as abstinence from alcohol is known to be one of the important prognostic factors of WKS patients. They are known to be at high risk of developing additional alcohol-related injuries and recurrent episodes if they do not remain abstinent [2]. Since CIWA-Ar consists of questions evaluating alcohol withdrawal symptoms rather than craving, our patient showed low score from the beginning.

This case study has several limitations. One is the fact that we could not make the use of functional neuroimaging studies, such as functional MRI and positron emission tomography which could have played an important role in guiding the application of rTMS. Second, we could not draw a conclusion regarding which specific cognitive domains are significantly improved after the rTMS. Hence we could not analyze the recovery pattern in different cognitive domains. Further studies will be needed to overcome these limitations and confirm its therapeutic efficacy.

To the best of our knowledge, this is the first report showing the efficacy of rTMS in the treatment of WKS. Although thiamine was administered immediately after the diagnosis and even after thiamine level was above the normal range, our patient did not show improvement in clinical symptoms. Considering the fact that he showed significant improvement after performing rTMS, we suggest rTMS has therapeutic efficacy in WKS by improving cognitive function, ataxia as well as decreasing alcohol craving at the same time.

\section{CONFLICT OF INTEREST}

No potential conflict of interest relevant to this article was reported.

\section{REFERENCES}

1. Thomson AD, Guerrini I, Marshall EJ. The evolution and treatment of Korsakoff's syndrome: out of sight, out of mind? Neuropsychol Rev 2012;22:81-92.

2. Kopelman MD, Thomson AD, Guerrini I, Marshall EJ. The Korsakoff syndrome: clinical aspects, psychology and treatment. Alcohol Alcohol 2009;44:148-54.

3. Guse B, Falkai P, Wobrock T. Cognitive effects of highfrequency repetitive transcranial magnetic stimulation: a systematic review. J Neural Transm (Vienna) 2010;117:105-22.

4. Bellamoli E, Manganotti P, Schwartz RP, Rimondo C, Gomma M, Serpelloni G. rTMS in the treatment of drug addiction: an update about human studies. Behav Neurol 2014;2014:815215.

5. Isenberg-Grzeda E, Kutner HE, Nicolson SE. Wernicke-Korsakoff-syndrome: under-recognized and under-treated. Psychosomatics 2012;53:507-16.

6. Nardone R, Holler Y, Storti M, Christova M, Tezzon F, Golaszewski S, et al. Thiamine deficiency induced neurochemical, neuroanatomical, and neuropsychological alterations: a reappraisal. ScientificWorldJournal 2013;2013:309143.

7. Brady KT, Gray KM, Tolliver BK. Cognitive enhancers in the treatment of substance use disorders: clinical evidence. Pharmacol Biochem Behav 2011;99:285-94.

8. Mishra BR, Nizamie SH, Das B, Praharaj SK. Efficacy of repetitive transcranial magnetic stimulation in alcohol dependence: a sham-controlled study. Addiction 2010;105:49-55.

9. Mishra BR, Praharaj SK, Katshu MZ, Sarkar S, Nizamie SH. Comparison of anticraving efficacy of right and left repetitive transcranial magnetic stimulation in alcohol dependence: a randomized double-blind study. J Neuropsychiatry Clin Neurosci 2015;27:e54-9.

10. Zhang G, Yao L, Zhang H, Long Z, Zhao X. Improved working memory performance through self-regulation of dorsal lateral prefrontal cortex activation using real-time fMRI. PLoS One 2013;8:e73735. 